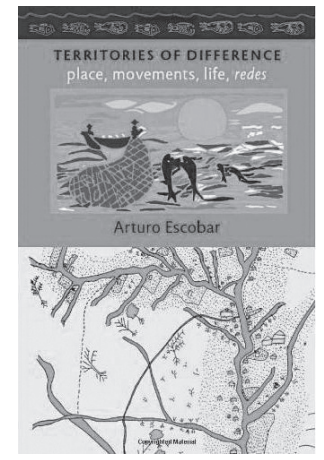

\section{Arturo Escobar. Territories of difference: place, movements, life, redes}

\author{
Durham, N. C.: Duke University Press, 2008, 435 p.
}

Manuel J. Prieto ${ }^{1}$
La corriente académica del postdesarrollo (post-development) se caracteriza por rechazar toda pretensión de naturalidad, neutralidad, o inocencia reclamada por las agendas desarrollistas que, impulsadas por las potencias económicas o por las distintas agencias internacionales, son impuestas y promovidas en el sur global. Es más, asumiendo una postura política, se rechaza toda imagen que presenta a aquellos países denominados desarrollados como ejemplos dignos a seguir por parte de los países subdesarrollados. Es dentro de este marco de referencia intelectual y político en el que se presenta el último libro de Arturo Escobar, profesor del departamento de Antropología de la Universidad de Carolina del Norte (Chapel Hill), titulado Territories of Difference: place, movement, life, redes.

Arturo Escobar constituye una referencia obligatoria dentro de los estudios del postdesarrollo, ello con especial énfasis en ámbito latinoamericano. Escobar, durante su carrera ha impulsado un original marco conceptual por medio del cual observa a las agendas pro desarrollo como una mera continuación de los proyectos colonialistas. Siguiendo esta idea, su trayectoria académica puede ser entendida como una doble invitación. En primer lugar, es una invitación a percibir el cómo la aplicación de los programas de desarrollo, más que beneficiosos, han generado una serie de daños en el sur global. En segundo lugar, Escobar invita a entender la narrativa del desarrollo bajo una mirada postestructuralista (Escobar, 1995). Así, predominantemente influenciado por la obra de Michael Foucault, Escobar observa que la idea de desarrollo está muy lejos de constituir un proceso natural y apolítico.
Por el contrario, el desarrollo y la agenda desarrollista forman parte de un discurso a partir del cual la idea de "Tercer Mundo" es una creatura que las agencias internacionales inventaron dentro del contexto de la guerra fría. En este sentido, el "Tercer Mundo," el "sur global," los "países en vía de desarroIlo," y otros conceptos afines, no serían más que construcciones sociales englobadas bajo la categoría de países subdesarrollados. Ello constituye un imaginario geográfico a partir del cual el primer mundo es investido de una natural autoridad moral e ideológica para intervenir con libre discreción en los destinos del Tercer Mundo. Dentro de este contexto, Territories of Difference es un libro en el cual Escobar aplica, expande y enriquece su aproximación teórica mediante el estudio etnográfico del movimiento social constituido por las comunidades afrocolombianas e indígenas del Pacífico colombiano (conocido como el "Proceso de las Comunidades $\mathrm{Ne}$ gras," o PCN) y su lucha por la defensa del territorio.

Escobar ha trabajado desde el año 1993 con los activistas del Proceso de las Comunidades Negras, siendo dicha experiencia su fuente de inspiración más importante para su trabajo sobre activismo, medio ambiente, cultura y desarrollo. Dentro de este proceso, el libro Territories of Difference viene a constituir el esperado corolario de tantos años de experiencia y trabajo etnográfico.

\footnotetext{
1 Escuela de Geografía y Desarrollo, Universidad de Arizona (Estados Unidos). E-mail: mjpm@email. arizona.edu
} 
El Pacífico colombiano es un perfecto caso de estudio para ilustrar la tensión clave explorada por los estudios del postdesarrollo. Aquí dos posiciones conviven en constante tensión, a saber: por un lado, las comunidades indígenas y afrocolombianas mantienen una cosmovisión única y particular que se refleja en un conocimiento local y encorporizado (embodied). Por otro lado, en el Pacífico colombiano también coexiste un proceso de acumulación capitalista el que, alegando universalidad y naturalidad, ignora el conocimiento local en nombre de la modernidad y el desarrollo de la zona. A partir de comienzos de la década de 1980, como parte de una agenda globalizadora, se impulsan una serie de planes y proyectos para constituir al Pacífico colombiano como una unidad territorial susceptible de ser desarroIlada. Es así como esta tensión entre formas de conocimiento se radicalizan, determinando la emergencia de movimientos locales que aspiran a la defensa del lugar, la naturaleza, y el conocimiento local. Aplicando un original y rico marco teórico, Escobar explora en detalles esta tensión destacando el cómo las diferencias y particularidades de los indígenas y los negros determinan la emergencia de un lugar único, entendido como una verdadera alternativa a la agenda desarrollista.

Si habría que reducir el libro de Escobar en cuatro palabras, diría que es un libro sobre la "política de la diferencia." Para Escobar, la idea de diferencia es el concepto clave para enfrentar el proceso mediante el cual lo moderno, el capitalismo, la globalización, y la visión occidental-eurocéntrica del mundo penetran al conocimiento y las prácticas de desarrollo local. Claramente inspirado por Deleuze y Guattari, Escobar ve en la idea de diferencia la única alternativa de escape ante la captura y reterritorialización ejercida por el Estado, el capital y los discursos de desarrollo. En este sentido, Territories of Difference explora el cómo, por un lado, el desarrollo y su proceso neocolonialista intenta homogeneizar (por ejemplo, borrar las diferencias) el conocimiento para así imponer una visión válida/oficial del mundo. Por otro lado, el libro expone las estrategias desarrolladas por los activistas locales para, por medio de la diferencia, resistir la penetración capitalista en sus prácticas de vida y cosmovisión. En definitiva, el libro presenta al concepto de diferencia como un alternativa descolonizadora ante la uniformidad territorial y epistemológica que el neocolonialismo capitalista pretende imponer bajo una pretensión de naturalidad y universalidad.

Advirtiendo la obsesiva prioridad que los estudios sobre globalización han dado al concepto de espacio (lo que aplica a la disciplina de la geografía), Escobar va a defender y destacar la utilización de la idea de lugar. Aquí, el lugar será analizado como socialmente construido, en el cual se articulan las diferencias y se resiste al proceso de globalización. Es por sobre la idea de lugar en donde activistas, indígenas, afrocolombianos, empresarios, agentes de gobierno, paramilitares, guerrilleros, agencias internacionales, intelectuales y otros entran en una dinámica de conflicto discursivo en la cual luchan por dominar la geopolítica del conocimiento. Ello, con el objetivo de materializar sus idearios de desarrollo y así construir un territorioregión que se acomode a sus intereses.

Desde un punto de vista metodológico, Territories of Difference representa una rica y novedosa descripción etnográfica de la dialéctica entre la diferencia de lo local y la homogeneidad del capitalismo global. En este sentido, Escobar se basa en el conocimiento generado por los propios activistas del Pacífico colombiano para desarrollar su análisis. Según Escobar, el libro "es una etnografía de las prácticas, estrategias, y visiones de este particular grupo de activistas, incluyendo su propia producción de conocimiento". La riqueza de la descripción privilegia las actividades de articulación por parte de quienes ejercen la defensa del lugar por sobre las actividades de la vida cotidiana. Por lo mismo, más que una etnografía de los afrocolombianos e indígenas, Territories of Difference es una etnografía de los movimientos sociales que se articulan en dicho lugar. Lo novedoso del ejercicio etnográfico presentado por Escobar, radica en que intenta romper con las barreras formales que separan al investigador del activista. Aquí, Escobar presenta lo que se podría denominar una "etnografía de la diferencia." Esto es, un trabajo etnográfico que, muy lejos de constituir a sujetos de estudio como meros objetos etnográficos uniformes, identifica el particular conocimiento producido por los activistas para aplicarlo a su marco 
de análisis. Siguiendo esta idea, Escobar va a exponer aquellas prácticas y conocimiento de carácter económico, social y cultural que permiten configurar la alternativa de lo diferente respecto al proceso homogeneizador controlado por la acumulación capitalista.

El enfoque principal del libro podría resumirse como un enfoque de "ecología política de la diferencia". Ello, en la medida que el autor reclama un reconocimiento para las ecologías, culturas, y economías locales como una real alternativa a lo moderno. Siguiendo con esta idea, el autor invita a desarrollar una ecología política antiesencialista, la cual se focalice en el proceso de producción de lugares particulares que, emergiendo desde particulares ensamblajes entre gente particular y ecosistemas particulares, se constituyan como plataformas subversivas de resistencia a la penetración capitalista.

El libro se organiza en seis capítulos más su debida introducción y conclusión. Cada capítulo explora con detalles y riqueza conceptual el cómo la idea de diferencia y particularidad se articula en el Pacífico colombiano a través de seis conceptos: lugar, capital, naturaleza, desarrollo, identidad y redes.

El primer capítulo es sobre el concepto de lugar. Este expone la emergencia del Pacífico colombiano como un territorio-región con identidad propia. Se destaca de qué manera las particularidades de dicho concepto se articulan como una verdadera barrera a los procesos de acumulación capitalista. En este sentido, el autor recalca la crítica feminista a la economía política de Gibson-Graham, a partir de la cual se sostiene el hecho de que los lugares nunca pueden ser completamente capturados por el capitalismo, la modernidad y la visión eurocéntrica del mundo. Por lo mismo, siempre conservan el potencial de convertirse en alternativas subversivas al desarrollo.

En el segundo capítulo, sobre capital, el autor explora el proceso a través del cual la acumulación capitalista se materializa en la forma de plantaciones de palmeras (para la producción de aceite) y granjas camaroneras. Ante este fenómeno, y sus impactos sociales y ambientales, Escobar expone las alternativas de resistencia que se han articulado. Ello, poniendo énfasis en el cómo distintas actividades productivas son desarrolladas por parte de las comunidades locales bajo dinámicas no capitalistas, entendidas como una economía diferente.

El capítulo tercero abarca el concepto de naturaleza. En este, el autor Ilama a explorar la tensa relación existente entre los distintos modelos de naturaleza que coexisten en el Pacífico colombiano. Escobar, además de entregar un rico debate teórico sobre las distintas aproximaciones epistemológicas a la idea de naturaleza, estimula a descolonizar las visiones dominantes de naturaleza y medio ambiente. Ello, por medio de una concepción que pone énfasis en la diferencia, en los discursos subalternos y en el conocimiento local.

En el cuarto capítulo Escobar lleva su argumento de la diferencia al ámbito del desarrollo. Aquí el autor critica las visiones universalistas y esencialistas de desarrollo, las cuales lo presentan como algo natural, universal, y como la única alternativa posible dentro de la senda moderna de globalización. En contra de esta visión, Escobar expone las alternativas que, desde la producción intelectual latinoamericana, entienden al proceso de desarrollo moderno como algo inseparable de la expansión colonial. Ello plantea la posibilidad de opciones descolonizadoras, las que, construidas desde la diferencia, demuestran la existencia de alternativas reales a la hegemonía moderna.

En el quinto capítulo, Escobar desarrolla el concepto de identidad. Aquí el autor destaca dicho concepto como la fuente básica a partir de la cual los movimientos indígenas y afrocolombianos articulan sus diferencias como una alternativa a la captura del Estado y el capital.

Por último el capítulo sobre redes presenta la mayor riqueza teórica del libro. Aquí Escobar expone el cómo las organizaciones sociales se organizan bajo las dinámicas de los sistemas emergentes-autoorganizativos.

Si bien es cierto el aparataje conceptual y teórico utilizado por Escobar a veces cansa, el libro da espacios para digerir la cantidad de información mediante resúmenes y clari- 
ficaciones. Posiblemente el lector instruido podrá encontrar algunas contradicciones e incoherencias dentro del marco conceptual, ello no le quita méritos al libro en la medida de que de todas forma su originalidad es fuente de inspiración y reflexión. Un problema importante del libro es la ausencia de un análisis de las relaciones de poder dentro del Proceso de las Comunidades Negras, privilegiándose una visión orgánica y cohesiva (casi esencialista) de los movimientos sociales. Otra debilidad es la ausencia de mayor profundidad etnográfica en la vida cotidiana de las comunidades afrocolombianas e indígenas. A pesar de que Escobar reconoce que es a partir de las acciones cotidianas de la vida que se articula la diferencia como forma de resistencia, el autor concentra su etnografía en los activistas.

En definitiva, Territories of Difference es una novedosa provocación a las formas en que pensamos el desarrollo; ello especialmente en contextos en donde las formas de conocimiento local aún resisten a la captura del neocolonialismo capitalista. Por lo mismo, el libro es una lectura obligatoria para quienes desde la geografía, antropología, sociología y disciplinas afines estén interesados en los movimientos sociales y la defensa del conocimiento local, el territorio y los recursos naturales. El rechazo y cuestionamiento que hace Escobar a la modernidad como una forma colonialista de imponer una visión universal y del mundo, hace del libro una importante referencia para quienes se interesen en los estudios de epistemologías subversivas como alternativas a las formas hegemónicas de producción de conocimiento. El libro también es una importante referencia para quienes estén interesados en explorar la aplicación de las ideas de redes, ensamblajes, flat ontologies, sistemas autoorganizativos, complejidad, entre otros, en entendimiento de los movimientos sociales, las relaciones humanomedio ambiente, y el pensamiento geográfico en general. Considerando la relevancia de estos temas en America Latina, urge una traducción de Territories of Difference al español y al portugués.

\section{Referencias bibliográficas}

ESCOBAR, A. Encountering development: The making and unmaking of the Third World. Princeton: Princeton University Press, 1995. 